\title{
Insurgencia de lenguas e identidad indígena en sistemas educativos una visión desde un país en vías de desarrollo
}

\author{
Languages Insurgency and Indigenous Identity in Education Systems: \\ A perspective from a developing country
}

María Fernanda Espinoza Cuesta ${ }^{1}$ Esteban Mauricio Inga Ortega ${ }^{2}$

\section{Resumen}

Se presenta los resultados que se obtuvieron al investigar los programas educomunicativos interculturales, en la importancia para la transformación del aprendizaje. Las estrategias de participación y colaboración que aportan al desarrollo individual y social, además de la integración multidisciplinaria. Reconociendo los espacios que ponen en práctica las políticas educomunicativas interculturales. En Ecuador, se dedican programas educativos desde el reconocimiento a sus 14 nacionalidades. Es decir, se espera que las reformas en los métodos educativos sean interculturales; Sin embargo, el kichwa al tener mayor número de hablantes después del lenguaje español, está ausente en las aulas y espacios de desarrollo social.

Representando una problemática para sus hablantes nativos. La limitación para poder acceder a una educación de calidad significa la ausencia de estudiantes en los distintos campos educativos. Por consiguiente, la pérdida del lenguaje kichwa e identidad de sus hablantes. Incrementando la desigualdad y falta de democracia. Los estudios muestran nuevas vías educomunicacionales para incorporar la interculturalidad por medio del ámbito virtual. De esta manera, se incrementa el acceso de estudiantes desde diferentes espacios, capacitando a educadores en la lengua kichwa como en métodos educativos interculturales. Las plataformas utilizan programas que se desarrollen en su lengua materna kichwa.

Palabras clave: Comunicación social; desarrollo; educación; interculturalidad; kichwa.

1 Estudiante de Comunicación social mención desarrollo, Universidad Politécnica Salesiana, Ecuador, mespinozac4@. est.ups.edu. ec http://orcid.org/oooo-0002-6782-6929

$2 \mathrm{PhD}$ en Ingeniería, Tutor de Seminario de Tesis, Universidad Politécnica Salesiana, Ecuador, einga@ups.edu.ec http://orcid. org/0000-0002-0837-0642

Recibido: 13/07/2020 - Aprobado: 05/10/2020 


\section{Abstract:}

The results obtained when investigating intercultural educommunicative programs are presented from the importance for the transformation of learning. Participation and collaboration strategies that contribute to individual and social development, in addition to multidisciplinary integration. Educational programs in Ecuador are dedicated from the recognition of their 14 nationalities, in other words, reforms in educational methods are expected to be intercultural; However, since Kichwa has a greater number of speakers after the Spanish language, it is absent in classrooms and spaces for social development.

The limitation of native speakers to be able to access a quality education means the absence of students in the different educational fields and, consequently, the loss of the Kichwa language and indigenous identity increases inequality and lack of democracy. Studies show new educommunicational ways to incorporate interculturality through the virtual environment. In this way, the access of students from different spaces is increased, training educators in the Kichwa language in intercultural methodology. The platforms use programs that are developed in their native Kichwa language.

Keywords: Social Communication; Development; Education; Interculturality; Kichwa Language.

\section{Introducción}

El presente artículo muestra como la educomunicación intercultural permite la transformación educativa por medio de estrategias de participación y colaboración interprofesional. Generando desarrollo individual y social; adicionalmente, responde necesidades diversas, adquiriendo un enfoque integral y multidimensional (Carm, 2013).

De igual manera, la democracia e igualdad son parte la educación intercultural. Mediante el respeto a la diversidad lingüística, se diseñan proyectos de inclusión de los lenguajes indígenas y ¿por consiguiente; el empoderamiento se dará en los distintos espacios sociales (Aikman, 1997).

La inclusión y participación permite la interacción de los hablantes nativos. Lipski (2014) se refiere a la integración de las lenguas confrontando a un idioma dominante, que limita la presencia de indígenas en diferentes sectores. Es el caso de la educación, no todos tienen el mismo acceso.

Restringiendo la oportunidad de desarrollo del sujeto Lehmann (2015) explica que, la educación se enfrenta a la enseñanza de personas de ascendencia indígena 


\section{EDUCACIÓN}

con el método de una segunda lengua; Sin embargo, la capacitación de educadores bilingües impulsara a una educación intercultural.

Por lo tanto, los maestros son más conscientes e incorpora un aula dinámica; Como resultado, no sólo los hablantes nativos participaran, sino, todos adquieren conocimiento de las distintas lenguas (Golemon, 2003).

Moya (1990) y Raybourn et al (2003), proponen el desarrollo intercultural apoyándose en la comunicación a través de medios audiovisuales. Aumentando el éxito educativo y dando como resultado la insurgencia; y por consiguiente, se utilizará una praxis descolonizante que no pretenda la desaparición de lo nativo (Jacob, 2017).

E. C. Moya y Molonia (2020) explican la importancia de una educación de calidad para la sostenibilidad e inclusión, al no existir esta se pierden la oportunidad de desarrollo. Una característica es el liderazgo inclusivo de las aulas, pues el espacio educativo se vuelve óptimo al contar con la participación de estudiantes y profesores.

Como se mencionaba anteriormente los docentes son base de fundamental para una mayor inclusión de las lenguas. Para que la integración de un modelo educomunicativo intercultural tenga éxito son necesarios los programas impartidos para maestros. Siendo competentes para dictar una materia intercultural bilingüe (Lova Mellado y Bolarín Martinez, 2015).

Ibañez-Etxeberria et al. (2020), (Recio y Santos, 2014) y (Hodam et al., 2020) plantean desde sus contextos la educación interculturalidad desde una plataforma virtual. Como se explicó anteriormente esta permite mayor oportunidad de acceso, también como explican los autores proporcionan a los estudiantes las capacidades en el ámbito tecnológico junto con el desarrollo intercultural lo cual permitirán un desenvolvimiento en la sociedad.

En la ilustración se expone la relación de una ciudad. En ella se pueden apreciar varias estructuras que representan a instituciones educativas. Seis de las siete instituciones brindan una educación bilingüe inglés/ español; Sin embargo, la única institución bilingüe de kichwa/español, se encuentra segregada en un espacio alejado y mostrando a un único estudiante. Refleja la dinámica del idioma dominante y la falta de instituciones educativas kichwa, programas que generen interés, estructuras de calidad (instalaciones), la participación de los estudiantes y maestros. 


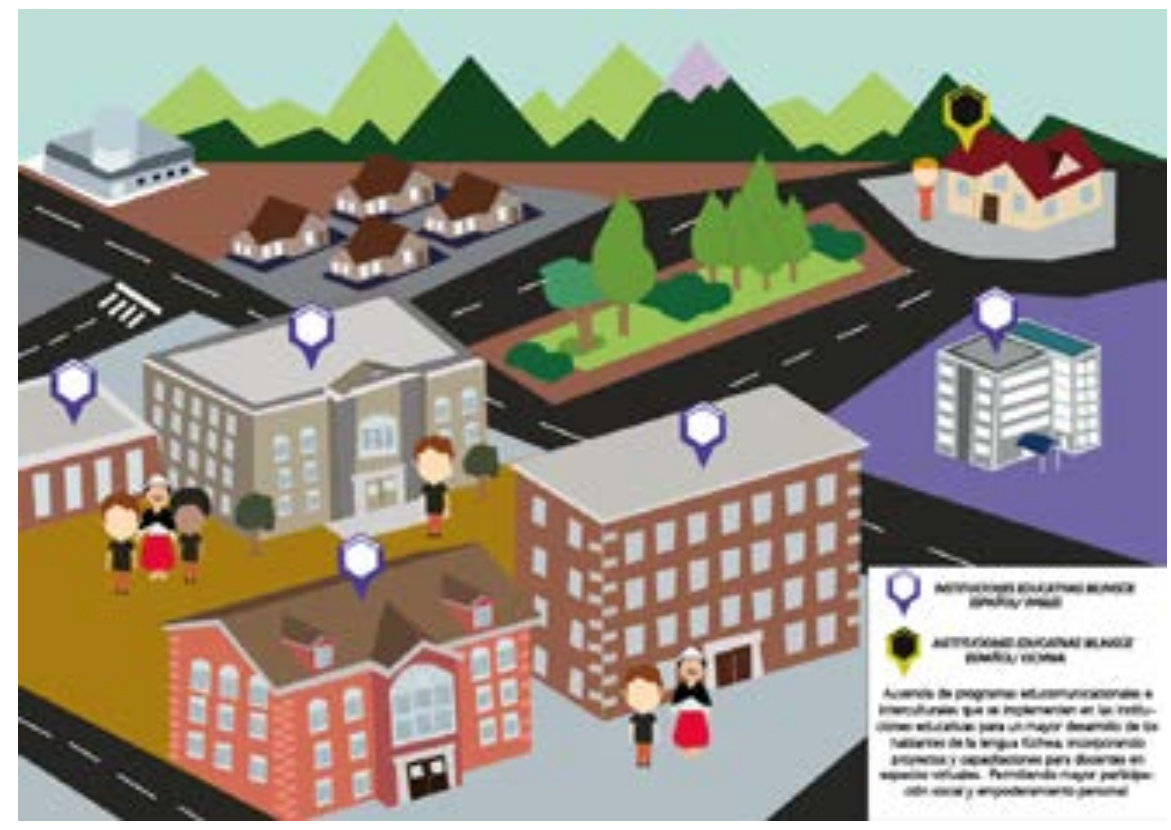

Ilustración 1. Fernanda Espinoza: Educomunicación e interculturalidad.

\section{Integración de lenguas a través de la educomunicación}

La interculturalidad pretende el diálogo entre las diferentes identidades culturales, dando a conocer las exigencias de los sectores históricamente marginados que buscan su integración en las sociedades, especialmente urbanas, en los diferentes campos que han sido segregados.

A través de la gestión de la comunicación en las diferentes áreas, la interculturalidad se considera como un nuevo método para el desarrollo social. Añazco (2019) se refiere a la interculturalidad como idea movilizadora que, surge de los procesos de la educación. La visibilización de los cambios permiten fortalecer las técnicas para una integración sin segregación.

Los cambios se producen desde la percepcion del sujeto, su comportamiento, relaciones interpersonales y conocimientos. Es entonces que, se cuestiona las estructuras dominantes y los sistemas (educativos). Se parte de reformular y reorganizar los espacios de flujo de comunicación (Añazco, 2019).

En estrecha relación comunicación/educación se reconfigura el desarrollo de inclusión. Añazco (2019) explica que, la educación será un motor de cambio, se deben considerar el potencial y nivel de alcance que tiene. Por lo tanto, su configuración dará a la educomunicación el fomento de la interculturalidad. 


\section{EDUCACIÓN}

La educación desde un nuevo constructo democrático de experiencias y conocimientos que tienden a ser relaciones circulares (homogeneidad). Constando de la participación e inclusión de todos los sujetos pertenecientes a una institución escolar; como resultado, se consolida una comunidad intercultural educativa (Darós, 2019) pero también autonomía o autodeterminación. Con una metodología propia de los ensayos, se ha considerado que la convivencia implica: a.

Es fundamental una educación de calidad con las condiciones adecuadas para la integración, con modelos flexibles que desarrollen a todos los estudiantes en los mismos niveles. Tomando en cuenta sus diferencias sociopolíticas se impulsarían sus relaciones interpersonales productivas, autonomía e impulsando el conocimiento (Darós, 2019)pero también autonomía o autodeterminación. Con una metodología propia de los ensayos, se ha considerado que la convivencia implica: a.

Propuestas educativas como en Argentina, donde se busca la inclusión de las minorías lingüísticas. Partiendo de comprender los contextos históricos y la constitución de las políticas educativas destinadas para la diversidad sociolingüística (Hecht, 2016).

Al existir mayor participación de las diferentes etnias se posibilita su inclusión en distintas áreas; consiguientemente, se visibilizan las tradiciones, costumbres y lenguas nativas. Hecht (2016) explica que la interculturalidad desde la política educativa en los países de Latinoamérica es un instrumento de empoderamiento; de hecho, aumenta la insurgencia de estos grupos en las aulas educativas.

Por lo tanto, es principal buscar alternativas de educación inclusiva, que critique a un sistema educativo tradicional dominante que, transgrede no sólo a estudiantes, sino a las personas provenientes de distintas realidades, para quienes su proceso de adaptación se vuelve una imposición.

Este mismo proceso sucede con los estudiantes que no provienen de pueblos indígenas puesto que su concepción del mundo se desarrolla a medida que se relaciona con los otros. Hecht (2016) hace referencia a la educación en sus primeros niveles educativos, donde la inclusión resulta un proceso más simple. Los estudiantes, si bien, provienen de contextos sociales diferentes no han sido manipulados en su totalidad con estereotipos sobre las comunidades indígenas.

El siguiente gráfico de la página Lens.org, es resultado de la búsqueda de las palabras comunicación social e interculturalidad. 


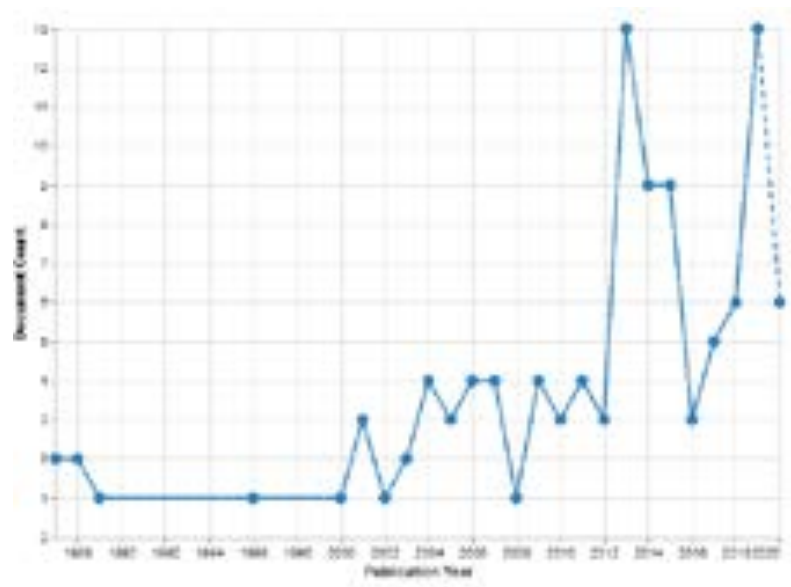

Ilustración 2: Lens.org, Año con alto índice de publicaciones.

Evalúa que tanto el 2013 y 2019 tienen un índice alto de publicaciones sobre los temas antes mencionados; Sin embargo, las publicaciones están aún muy por debajo de lo esperado al tratarse de problemas globales de integración en distintos campos; por otra parte, se destaca los sistemas educativos tradicionales que desde los primeros años genera sentimientos de individualidad, dando como resultado crisis en las relaciones socioculturales (Cherkashina et al., 2019).

Estas relaciones de direccionan a la percepción del "propio - extraño", con la supuesta "tolerancia"; de hecho, el factor de la barrera lingüística, imposibilita lo antes mencionado, el empoderamiento. Puesto que se dan conflictos y no permite un correcto desarrollo.

El desconocimiento de las lenguas lleva a la segregación de modo cultural y territorial. Por esta razón, en Chile se interesan en la integración de los hablantes de otras lenguas. Generando interés en los lingüistas, que a través de estudios proponen objetivos interculturales. Conocer realidades (escritura/sonidos de las lenguas), educación integral lingüística y aplicación del enfoque comunicativo intercultural (Souza y Salgado, 2020).

Chile trabaja con la política educativa lingüística desde 1996, con el programa de Educación Intercultural Bilingüe; Adicionalmente, se compone de la competencia comunicativa intercultural. Visibilizando habilidades cognitivas, afectivas y actitudinales de los hablantes nativos. De este modo reconoce y legitima a las lenguas indígenas, promueve el aprendizaje de una segunda lengua indígena (Souza y Salgado, 2020).

Capacitando a todos los estudiantes de un nivel alto de competencia intercultural, preparándolos para un mejor desarrollo social, preparados para actuar a favor de sus 


\section{EDUCACIÓN}

comunidades generando flujos de comunicación comprensibles, permitiendo reconocer las necesidades de los pueblos (Souza y Salgado, 2020).

De la misma manera en Perú, se considera la transformación que tienen las sociedades por varios factores (migración). Implicando la inserción de servicios interculturales bilingües para sus poblaciones quechua, aymara y amazónica. Dando espacio a que los hablantes continúen su educación en sus lenguas maternas (García-Segura, 2019).

Espinosa (2017) propone que cada uno de los países generen estudios sobre las posibilidades económicas de sus estudiantes ante la posibilidad de acceder a una educación de calidad que particularmente es clásica, represiva y no prioriza una buena comunicación para compresión de los tópicos.

Por esto es importante comprender que la interculturalidad y acceso a la educación de inclusión, no significa imponer la lengua dominante al hablante indígena; de hecho, se pretende que las instituciones cambien los sistemas educativos tradicionales (etnocentrista), promoviendo procesos de descolonización.

Como se menciona anteriormente, la primera fase donde se aplica la educación intercultural es con los niños, quienes acompañados de docentes que están formados, capacitados, disponen de materiales e impulsan una educación de calidad (GarcíaSegura, 2019).

La aplicación de educación intercultural bilingüe está integrada de varios elementos que legitiman la aplicación de estrategias metodológicas. Primer elemento, los espacios educativos que son parte de la realidad cotidiana, su reestructuración, nueva distribución de la ubicación dentro del aula que permitan una mejor socialización (Tenorio Sánchez, 2019).

Esta estrategia desde el campo psicológico permite mayor participación y los indígenas desde su cosmovisión y percepción del aula de clase consideran que, la nueva distribución (circular) genera mayor comunicación, es decir, se forma cada estudiante en las mismas condiciones, crecimiento de interacción y empoderamiento de la cultura y que no solo nativos (Tenorio Sánchez, 2019).

La recuperación de espacios educativos permite la reconstrucción de la memoria, experiencias, vivencias, recuperación de autoridad, valoración del lenguaje, etc. Fortaleciendo el pensamiento crítico, el mismo es fundamental sea impulsado por maestros, científicos e investigadores.

Al recordar los procesos educomunicativos se comprende cuáles han sido los retos para enfrentar en la enseñanza. Es el caso de Ecuador, que actualmente reconoce sus 14 nacionalidades, aun así, en el pasado sus comunidades indígenas fueron perseguidas y 
asesinadas por enseñar su lengua materna, además de no tener acceso a la educación (González Terreros, 2015).

La educación intercultural desde un diálogo horizontal con el objetivo de inclusión, pretende recuperar la diversidad del pensamiento de cada individuo. De hecho, pone en práctica otro elemento, la comunicación intercultural, a través de diferentes medios. Para el diseño de estos canales, se involucra profesionales capacitados y que promuevan las relaciones positivas entre grupos culturales (Montoya Ortega \& Paiz Malespín, 2018).

Recordemos que existen diferentes canales de comunicación donde se puede desarrollar la interculturalidad; sin embargo, se toma en consideración aquellos que además de educar generen relaciones interpersonales, con ambientes propicios donde se genere la participación. Puesto que es una educación abierta y con un modelo que rompe la estructura clásica (Bacquet-Pérez y Batres-Quevedo, 2019).

Este nuevo modelo procurar que no existan manifestaciones de discriminación, racismo o exclusión por y consiguiente, los medios de comunicación desde su diseño deben estar compuestos por información que fomente buenas relaciones, proporcionando la misma educación para cada estudiante (equidad e igualdad), beneficiando la integración (actividades) y el enriquecimiento mutuo (compartir saberes) (Montoya Ortega y Paiz Malespín, 2018).

Los espacios de comunicación que se aplicarán para el mejor desarrollo de la educación intercultural pueden ser de forma física o virtual, puesto que estas ramas se apoyan en las nuevas tecnologías que, además de permitir una mayor comunicación, también brindan mayor acceso a información.

Colombia desde su política intercultural, visualizando el desplazamiento desde zonas rurales a capitales y el incremento de la tecnología, desarrollan estrategias educomunicacionales que permitan tener encuentros interculturales. Aplican programas virtuales que no tienen limitación para ninguna población cultural. (Barreto y Rivilla, 2013).

El maestro continúa siendo un elemento importante para mantener la interacción correcta en estas plataformas. Barreto y Rivilla (2013) se refieren a que los maestros deben recibir capacitación sobre la historia, cultura, tradición y lingüística; consideran que aún existe la incapacidad de identificar estrategias adecuadas para la inclusión cultural.

Es primordial que todos los campos de la educación trabajen en un conjunto intercultural, solo de ese modo se pueden cumplir objetivos. En el ámbito virtual 


\section{EDUCACIÓN}

tanto el interés del maestro como los estudiantes es fundamental para que se generé un cambio educativo.

Por lo cual, la percepción ante la mejora lingüística se debe dar por todos los medios de comunicación que permitan una práctica, tanto oral como escrita; a diferencia de la concepción habitual sobre el aprendizaje de una lengua como único elemento de superación individual(Guadamillas Gómez, 2017).

Los objetivos son la integración de sujetos que hablen distintas lenguas y no tengan que ser segregados (obligados a dejar su lengua materna), sino, tengan la posibilidad de desempeñarse sin limitaciones. Guadamillas Gómez (2017) se refiere a que los alumnos (lengua minoritaria) cuando se encuentra en el aula tienen carencias léxicas, vocabulario sencillo y escasez de recursos conversacionales lo cual les produce inseguridades o ansiedad.

Es importante la reconstrucción del espacio educativo, diseño de programas que capaciten a los educadores de manera propicia con herramientas educomunicacionales, generando el interés en los estudiantes y motivando su participación e interés ante diversidad de las culturas; además de generarse un proceso de empoderamiento en los hablantes nativos que, tienen más espacios de participación y no estarán sometidos a un sistema educativo tradicional.

\section{Formulación del problema y metodológica}

La lengua kichwa es parte de los 14 idiomas reconocidos en la Constitución de la República del Ecuador. Sin embargo, en la zona urbana se carece de espacios donde tanto sus hablantes o interesados puedan desarrollarse con comodidad. Estas terminan siendo limitantes y se contradicen con la Constitución que, al reconocerla estas entidades e instituciones estarían capacitadas para atender a los hablantes. Pero, esta falta de espacios no es un problema únicamente de un no reconocimiento.

Al mirar más a fondo encontramos problemas sociales como estereotipos alrededor de la lengua kichwa, una degradación al indígena, heredado desde tiempos de la colonia. Atiborrada de connotaciones degradantes. Hablada por un pueblo usurpado, señalado como ignorante, sucio y salvaje. Esos son prejuicios que han perdurado durante décadas como regalo del colonizador; sin embargo, en los pueblos indígenas la insurgencia de sus tradiciones y costumbres, han permitido que se mantenga la lengua kichwa.

Estos hablantes nativos con los años se mudan a las ciudades grandes donde el lenguaje dominante es el español. Obligados dejan de hablar su idioma, porque la urbe carece de interés para aprender esta lengua. Los hablantes de kichwa se mudan con su familia y poco a poco son sus hijos que asisten a escuelas en español, van dejando su 
lengua materna. De esta problemática se percata el Sistema de Educación y propone La Educación Intercultural. Compuesta de programas que buscan la integración de las culturas y salvaguardar las lenguas. La aplicación se dio con gran interés, pero con los años se perdieron los espacios de enseñanza en kichwa.

En Quito existe únicamente una escuela bilingüe de kichwa- español, ubicada en San Roque. La falta de programas educomunicacionales, traerá problemas a largo plazo. La pérdida de hablantes nativos, como sucede con el zápara con solo 3 hablantes; adicionalmente, los hablantes nativos al enfrentarse a una lengua dominante se enfrentan a problemas internos.

El problema que he establecido en base a mi investigación parte de la educación, ya que es el ámbito donde se aprende, forma y moldea a una persona. Como es necesaria la implantación de programas educomunicativos que capaciten a profesores y alumnos para desarrollarse de manera adecuada en un aula intercultural que en este caso es virtual por su mayor posibilidad de acceso.

Dar a conocer esta problemática como estudio, permitirá conocer los procesos que posibilitan reforzar la educación intercultural, con el soporte de la comunicación, que impulsa su aplicación en distintos canales. Proporcionando de herramientas para la creación estrategias que permitan el cumplimiento de los objetivos.

El método aplicado en el artículo de investigación es el experimental, ya que permite recopilar información. Por lo cual se ha generado una hipótesis sobre la educomunicación intercultural y las posibilidades de desarrollo social. Se plantea que, la educación intercultural bilingüe genera ambientes de comunicación adecuada para un desarrollo de sostenibilidad propicio para los hablantes.

En principio se utilizará la herramienta de software Vosviewer para identificar cuáles son los países donde se realizan más investigaciones relacionadas a la temática, ya que este software permite conocer los trabajos de investigación que se realizan alrededor del mundo. Para una mejor visión de los países con mayor estudio sobre la temática se realizará una comparación entre dos gráficas Vosviewer, pero de la página Scopus y Web of Science.

La herramienta para identificar las relaciones y redes existentes es el software ATLAS.TI versión 8, con el cual podremos conocer cuáles son las palabras que se encuentran relacionadas en cada uno de los artículos analizados, a través de una nube de palabras. Al mismo tiempo que el software permite establecer códigos que por medio de una red permitirá conocer de mejor manera las relaciones existentes entre los artículos. 


\section{Análisis de los resultados}

A continuación, la red de interacción es tomada de la página Scopus. Se analizó comunicación y educación.

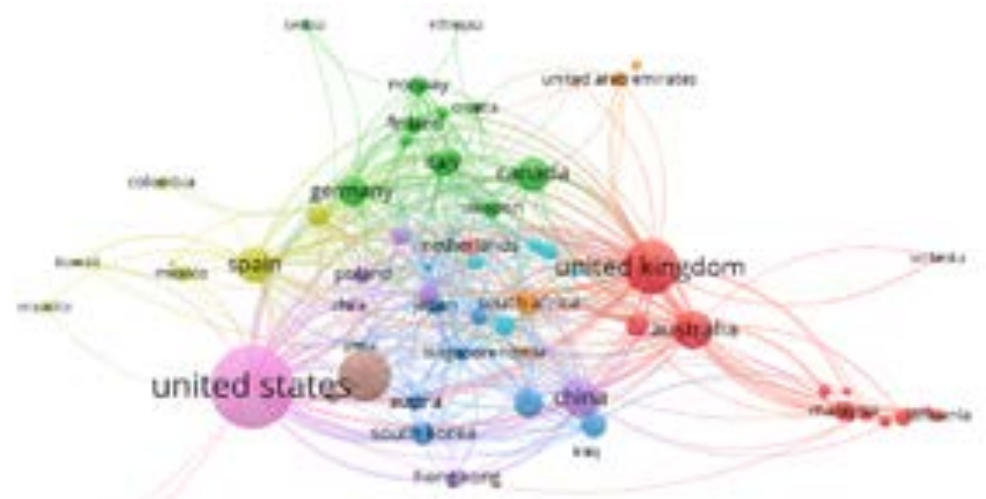

Wosviener

Ilustración 3: Scopus, Red de comunicación y educación, 2020

Como se visualiza el país de mayor relevancia e influencia es Estados Unidos, conectado con Reino Unido, Canadá, Alemania, Italia y países que con menos influencia forman parte de esta red (Ecuador- México -Colombia).

De la misma manera, la siguiente gráfica de red de la página de indexación Web of Science, se revisó la misma temática anterior.

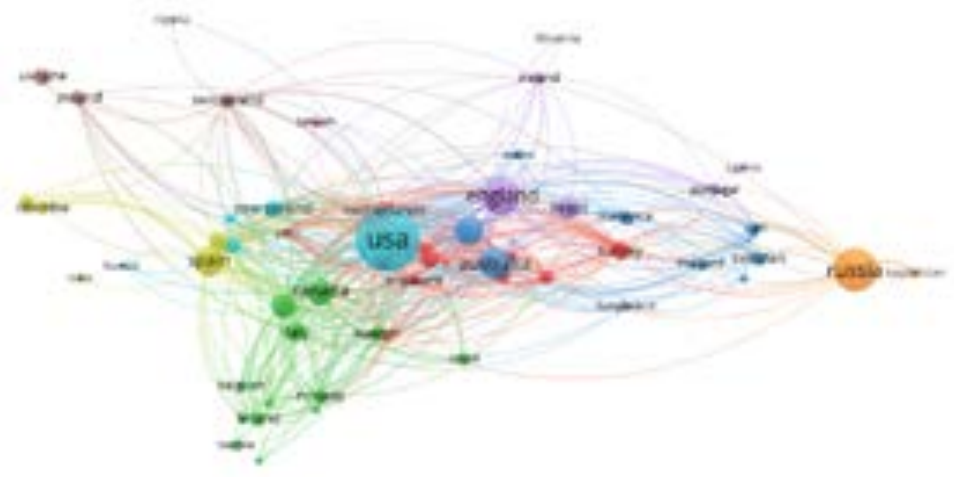

A. vostiene

Ilustración 4: Web of Science, Red de comunicación y educación, 2020 
El gráfico anterior Estados Unidos tiene mayor relevancia; Sin embargo, aparecen otros países que en el otro gráfico no tenían la misma cantidad en relevancia (RusiaAustralia- Inglaterra- España). En este caso los países que desparecen en comparación al anterior grafica se encuentran México, Ecuador, aun así, se mantiene Colombia.

Estas muestran la importancia de la investigación para la aplicación de nuevos sistemas de educación, de modo que se integra la visión desde distintas realizas; entonces, surge la necesidad de comprender las necesidades, carencias y elementos que componen este nuevo modelo de educación.

En la nube de palabras del software ATLAS.TI encontramos que la relación de las palabras entre los artículos analizados es la siguiente:

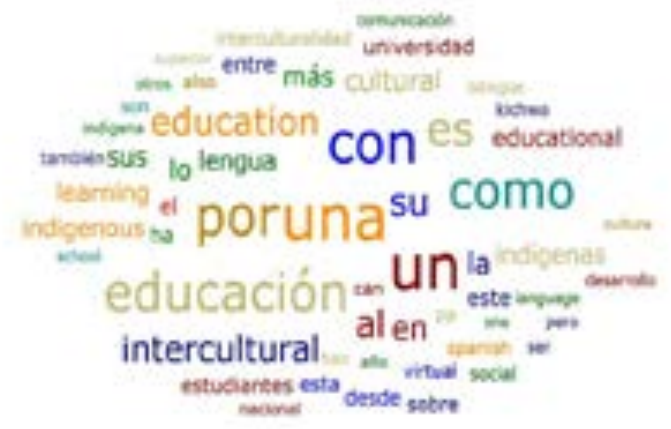

llustración 5: ATLAS.TI Nube de palabras, 2020.

Podemos constatar que en los artículos analizados las palabras de nuestro tema de investigación se encuentran presentes como lo es educación, interculturalidad, lengua, cultura, comunicación, desarrollo.

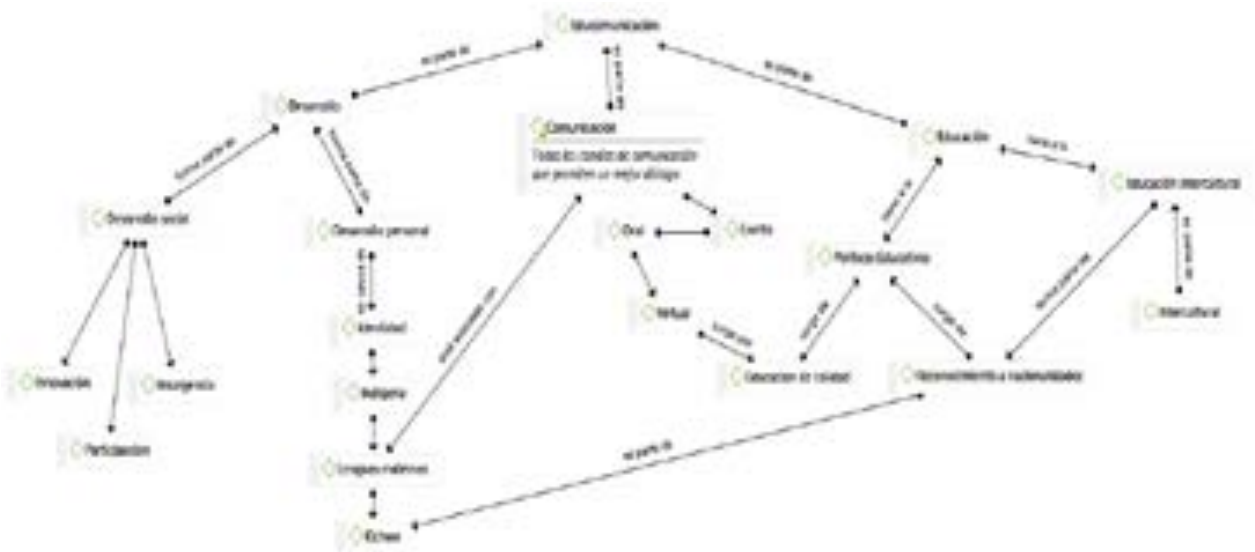

llustración 6: ATLAS.TI Red de artículos, 2020. 


\section{EDUCACIÓN}

En esta gráfica encontramos las relaciones planteadas bajo una red de conexiones de los temas que predominan en las lecturas. Dejando ver qué desarrollo, comunicación y educación son parte de la educomunicación.

La siguiente red de ATLAS.TI muestra como las relaciones se dan en torno a la interculturalidad y como se generan diferentes conexiones.

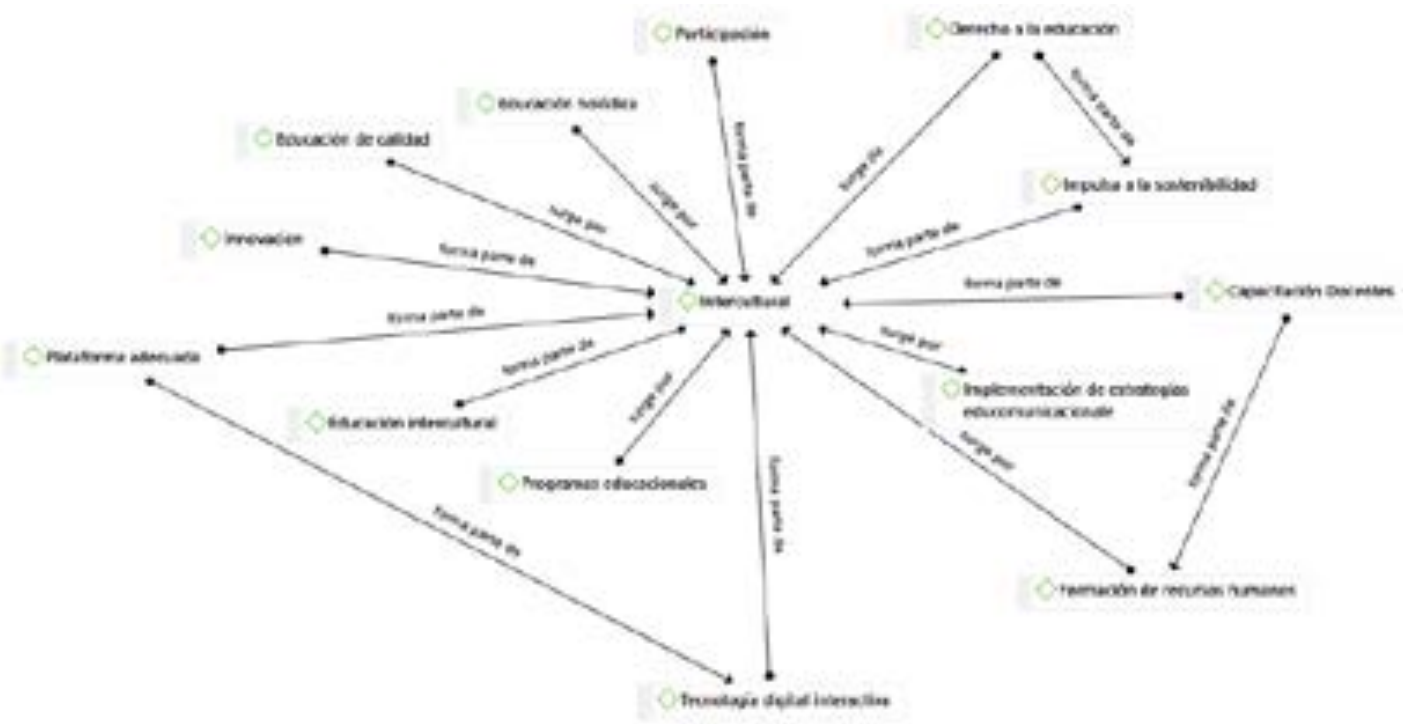

llustración 7: ATLAS.TI Red intercultural, 2020.

\section{Conclusiones}

La investigación de este articulo planteó como hipótesis que la implantación de una educomunicación intercultural permite un mayor desarrollo sostenible en los sujetos hablantes de las distintas lenguas, como podemos constatar en las gráficas de ATLAS.TI existen conexiones que se complementan para generar mayores posibilidades de acceder con derechos a una educación de calidad con docentes capacitados.

La mayoría de investigaciones relacionadas a la temática se las realiza en países del primer mundo, pues estos también buscan la mejor implementación de la educación bilingüe en sus sistemas; Sin embargo, los países latinoamericanos que cuentan con el mayor número de hablantes de kichwa no aparecen como focos de estudio en las gráficas de Vosviewer.

Se expone entonces la necesidad de implementar sistemas educomunicativos en los sistemas educación dejando atrás las limitaciones clásicas que no permiten la insurgencia de los pueblos, lo cual lo excluye. En la investigación se pudo detectar 
que existen planes desarrollándose en distintos países de Latinoamérica, los cuales se enfocan en generar espacios de diálogo.

La comunicación es entonces la mejor vía para impulsar la educación intercultural, pues permite por distintos canales expresar las ideas y tener mayor compresión. Esta al relacionarse con otras disciplinas impulsara la eficacia de la educación, tomando en cuenta los nuevos contextos (digital) que permiten tener mayor alcance en los estudiantes.

\section{Agradecimiento}

Esta publicación obtuvo el financiamiento de: El Fondo de Asistencia Internacional de los Estudiantes y Académicos Noruegos (SAIH).

\section{Lista de referencias}

Aikman, S. (1997). Interculturality and Intercultural Education: A Challenge for Democracy. Tradition, Modernity and Post-Modernity in Comparative Education, 43, 463-479. https://doi.org/10.007/978-94-011-5202-0-6

Añazco, D. (2019). La interculturalidad como nuevo objeto: Esbozo de un planteamiento transmoderno. Ciencia e Interculturalidad, 24(01), 106-119. https://doi. org/10.5377/rci.v24io1.80o6

Bacquet-Pérez, C. N. O., \& Batres-Quevedo, J. A. (2019). Animales tejidos y educación ambiental en comunidades kichwas de Tena. Killkana Social, 3(2), 21-28. https:// doi.org/10.26871/killkana_social.v3i2.466

Barreto, C. R., \& Rivilla, A. M. (2013). Actitudes y creencias de la competencia intercultural en profesores virtuales. Ingeniería y Desarollo, 31(2), 272-29o.

Carm, E. (2013). Rethinking education for all. Sustainability (Switzerland), 5(8), 3447-3472. https://doi.org/10.3390/su5083447

Cherkashina, T., Novikova, N., Dembitskyi, S., Pugachev, I., y Iakovleva, S. (2019). La lengua al servicio de la adaptación sociocultural y económica de los migrantes en la época de las transformaciones globales. Andamios Revista de Investigación Social, 16(39), 283. https://doi.org/10.29092/uacm.v16i39.684

Darós, W. R. (2019). Ensayo sobre la educación como convivencia para superar las esci-

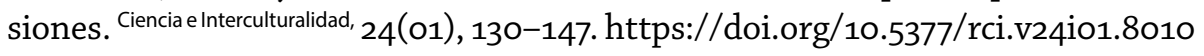




\section{EDUCACIÓN}

Espinosa, O. (2017). Educación superior para indígenas de la Amazonía peruana : balance y desafíos. Anthropologica, 39, 99-122. http://dx.doi.org/10.1880o/ anthropologica.201702.005

García-Segura, S. (2019). Identidad, lengua y educación: la realidad de la amazonía peruana. Revista de Estudios y Experiencias En Educación, 18(36), 193-207. https:// doi.org/10.21703/rexe.20191836garcia1

Golemon, P. (2003). Communicating in the Intercultural Classroom. IEEE Transactions on Professional Communication, 46(3), 231-235. https://doi.org/10.1109/ TPC.2003.816786

González Terreros, M. I. (2015). Las escuelas clandestinas en Ecuador. Raíces de la educación indígena intercultural. Revista Colombiana de Educación, 1(69), 75-95. https://doi.org/10.17227/01203916.69rce75.95

Guadamillas Gómez, M. V. (2017). Los intercambios virtuales lingüísticos y culturales en educación superior: un estudio de caso TT - Language and culture virtual exchanges in higher education: A case study. Apertura (Guadalajara, Jal.), 9(1), 8-21. https://doi.org/10.18381/Ap.v9n1.1016

Hecht, A. C. (2016). Educación Intercultural Bilingüe En Argentina: Un Panorama Actual. Ciencia e Interculturalidad, 16(1), 20-30. https://doi.org/10.5377/rci. v16i1.2351

Hodam, H., Rienow, A., y Jürgens, C. (2020). Bringing earth observation to schools with digital integrated learning environments. Remote Sensing, 12(3), 1-19. https://doi.org/10.3390/rs12030345

Ibañez-Etxeberria, A., Gómez-Carrasco, C. J., Fontal, O., \& García-Ceballos, S. (2020). Virtual environments and augmented reality applied to heritage education. An evaluative study. Applied Sciences (Switzerland), 10(7). https://doi.org/10.339o/ app10072352

Jacob, M. M. (2017). Indigenous studies speaks to American sociology: The need for individual and social transformations of Indigenous education in the USA. Social Sciences, 7(1), 1-10. https://doi.org/10.339o/socsci7010001

Lehmann, D. (2015). Convergencias y divergencias en la educación superior intercultural en México. Revista Mexicana de Ciencias Politicas y Sociales, 6o(223), 133-170. https://doi.org/10.1016/So185-1918(15)72133-1 
Lipski, J. M. (2014). Syncretic discourse markers in Kichwa-influenced Spanish: Transfer vs. emergence. Lingua, 151(PB), 216-239. https://doi.org/10.1016/j. lingua.2014.07.002

Lova Mellado, M., \& Bolarín Martinez, M. J. (2015). Coordination in bilingual programs: TeacherRsingle voices. Aula Abierta, 43(2), 102-109. https://doi.org/10.1016/j. aula.2015.03.001

Montoya Ortega, Y., \& Paiz Malespín, G. (2018). La comunicación intercultural en el aula de clases: Un estudio con estudiantes de Psicología en Contextos Multiculturales. Ciencia e Interculturalidad, 23(2), 46-58. https://doi.org/10.5377/rci.v23i2.6567

Moya, E. C., \& Molonia, T. (2020). Inclusive Leadership and Education Quality: Adaptation and Validation of the Questionnaire "Inclusive Leadership in Schools" ( LEI-Q) to the Italian Context. 12. https://doi.org/10.339o/su12135375

Moya, R. (1990). A decade of bilingual education and indigenous participation in Ecuador. Prospects, 20(3), 331-343. https://doi.org/10.1007/BFo2195075

Raybourn, E. M., Kings, N., \& Davies, J. (2003). Adding cultural signposts in adaptive community-based virtual environments. Interacting with Computers, 15(1), 91-107. https://doi.org/10.1016/So953-5438(02)00056-5

Recio, J. C. M., \& Santos, J. A. (2014). Modelo integrador para la formación de profesionales de la comunicación en entornos virtuales: preparando emprendedores. Investigacion Bibliotecologica, 28(64), 75-100. https://doi.org/10.1016/ s0187-358x(14)70910-4

Souza, L. L. de, \& Salgado, R. G. (2020). Narrativas sobre los Procesos de Inclusión y Exclusión de las Disidencias Culturales, Étnicas, Políticas, de Edad, Sexuales y de Género en la Educación Escolar en Chile y Brasil. Revista Latinoamericana de Educación Inclusiva, 14(1), 17-20. https://doi.org/10.4067/ so718-73782020000100017

Tenorio Sánchez, D. E. (2019). El Camino de Resintencia Cultural del Pueblo Ampiuile: Una Pedagogía de Pervivencia desde el Proceso Educativo Propio, Territorio Indígena Ampiuile, Municipio de Silvia Cauca, Colombia. Ciencia e Interculturalidad, 25(2), 9-22. https://doi.org/10.5377/rci.v25i2.8539 